\title{
NOVOS ANTAGONISTAS DA ENZIMA 3-HIDRÓXI-QUINURENINA TRANSAMINASE DE Aedes aegypti: SÍNTESE, ESTUDOS DE DOCKING MOLECULAR E ENSAIOS ENZIMÁ TICOS
}

\author{
Larissa Dias da Silva ${ }^{1 *}$, Denys Ewerton da Silva Santos ${ }^{1}$, Janaína Versiani dos Anjos ${ }^{1}$, Thereza Amélia Soares ${ }^{1}$ \\ ${ }^{1}$ Departamento de Química Fundamental, UFPE. \\ *Larissa.dias@ufpe.br
}

\section{INTRODUÇÃO}

O mosquito Aedes aegypti é o vetor primário responsável pela transmissão dos vírus zika, chikungunya, dengue e febre amarela. ${ }^{1}$ 0 controle do mosquito é uma tarefa árdua devido à sua grande adaptabilidade à ambientes urbanos, alto comportamento antropofílico, além de outros fatores biológicos. Além disso, o desenvolvimento de vacinas contra todas as arboviroses e seus mú Itiplos sorotipos tem um custo elevado e requer um longo período de desenvolvimento e testes em pacientes. ${ }^{2}$ Portanto, a alternativa mais eficaz para controle do inseto vetor permanece sendo o desenvolvimento e uso de inseticidas com diferentes modos de açã o. ${ }^{3}$ Estes novos inseticidas devem apresentar alta especificidade para a espécie alvo e baixa toxicidade para animais de grande porte, em especial mamíferos. ${ }^{3}$ Uma via metabólica importante em mosquitos é a via das quinureninas, responsável pela desintoxicaçã o de espécies reativas de oxigênio e nitrogênio em insetos (Figura $1)$.

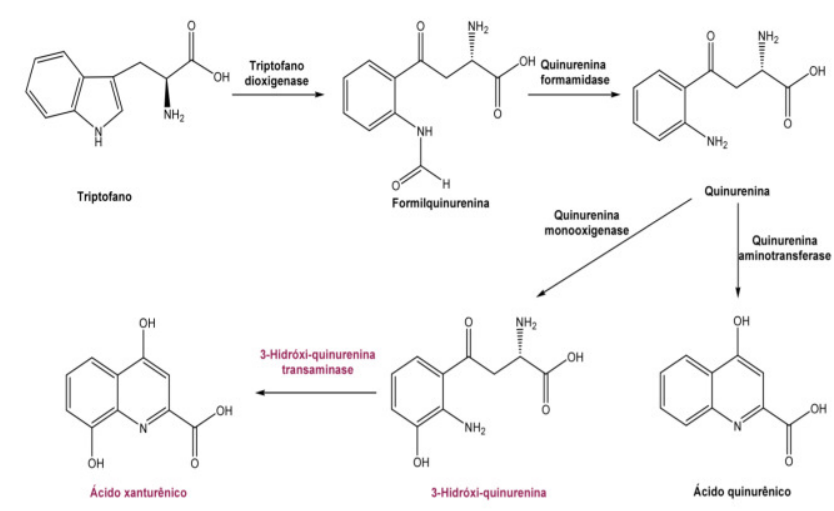

Figura 1 - Via das quinureninas em mosquitos.

A via das quinureninas é também a principal via de degradação do triptofano em organismos vivos, onde o triptofano é oxidado a quinolínico, ácido quinurênico ou a ácido xanturénico, dependendo do organismo estudado..$^{4-6}$ Mecanismos que levem ao acúmulo de espécies reativas de oxigênio e nitrogênio em insetos constituem uma poderosa arma no combate ao Ae. aegypti. O acúmulo de um dos intermediários da via das quinureninas, a 3-hidróxiquinurenina, leva à formação de "radicais livres" (espécies reativas de oxigênio e nitrogênio), o que resulta em apoptose das células neuronais e distúrbios motores nos insetos. A 3-hidróxi-quinurenina (3-HK) provém da quinurenina e leva à formação do ácido xanturê nico. Este último metabólito é atóxico para o inseto, provando que a reação que leva à sua formação consiste numa via de desintoxicação da 3 -HK nos mosquitos. ${ }^{4,5}$ Portanto, o uso de antagonistas competitivos da enzima 3-hidróxi-quinurenina transaminase (HKT) inibe a conversão da 3-HK em ácido xanturê nico, gerando acúmulo da 3-HK, potente agente neurotóxico para o A. aegypti.

Recentemente, os nossos grupos de pesquisa sintetizaram e avaliaram a atividade inibidora de ácidos de 1,2,4-oxadiazol contra a enzima HKT. ${ }^{7}$ Os cálculos de docking molecular indicaram que estes compostos heterocíclicos poderiam se ligar a HKT de forma análoga ao inibidor cristalográfico. ${ }^{7}$ Estes compostos foram testados utilizando larvas de quarto estádio de A. aegypti. Verificou-se que os efeitos da substituição do anel heterocíclico nã o eram tão relevantes, mas os ácidos e ésteres contendo halogê nios no anel aromático presente em $\mathrm{C}-3$ do heterociclo eram mais potentes. ${ }^{7}$ No entanto, estes compostos requerem co-solventes para serem solubilizados, o que dificulta a sua utilização como larvicida. Ainda recentemente, o nosso grupo mostrou ser possível a expressão da HKT de $A$. aegypti recombinante em Escherichia coli, e a obtenção além de novos derivados de ácidos butanóicos de 1,2,4-oxadiazol solúveis em água com resultados promissores frente à inibição da $\mathrm{HKT}^{8}{ }^{8} \mathrm{~A}$ partir da expressão da enzima em $E$. coli em grande quantidade, iniciamos a realização de ensaios enzimáticos e experimentos de cristalização da HKT para obtenção de estrutura cristalográfica (apenas a estrutura 3D da HKT de An. gambiae está disponível na literatura). 0 presente projeto busca expandir a coleção de compostos 1,2,4-oxadiazol solúveis em água através da síntese dirigida por cálculos de docking molecular, e mais especificamente, simulações de metadinâmica para estimar o perfil de enregia livre de ligação dos novos compostos a enzima HKT. Estes cálculos serão futuramente validados por ensaios enzimáticos da capacidade inibitória destes compostos contra a enzima HKT de $A e$. aegypti recombinante expressa em sistema heterólogo.

\section{MATERIAIS E MÉTODOS}

A etapa inicial do projeto, apresentada neste trabalho, é o desenvolvimento de modelos atomísticos computacionais da HKT e dos derivados de ácidos butanóicos de 1,2,4-oxadiazol de modo a observar através de dinâmica molecular como se desenvolve a interação entre essas moléculas e qual derivado tem a interação 
mais efetiva de modo a ser classificado como potencial substituto competitivo para o inibidor cristalográfico da enzima HKT. O modelo de água utilizado nas simulações foi o SPC/E. O algoritmo utilizado nas etapas de minimização de energia foi o Steepest Descent. 0 algoritmo foi empregado até que a maior força encontrada sobre as partículas do sistema tenha menor que 10 $\mathrm{kJmol}^{-1} \mathrm{~nm}^{-1}$. 0 raio de corte utilizado para otimizar o cálculo das interações eletrostáticas e de van der Waals foi de 1,4 $\mathrm{nm}$. Nas simulações, as quais fizeram uso do campo de reação como tratamento eletrostático de longa distância, foi utilizado um valor de 66,6 para a constante dielétrica do campo de reação ${ }^{19}$. 0 algoritmo de restrição LINCS foi utilizado a fim de manter o comprimento das ligações dos solutos e da geometria das molé culas de água constantes. Com a aplicação dessa metodologia, foi possível utilizar um passo temporal para as dinâmicas de $2 \mathrm{fs}$. 0 acoplamento foi realizado separadamente para diferentes grupos. Nas simulações em água, os grupos foram representados pela proteína e pelo solvente com adição dos íons. A pressão do sistema foi mantida constante, através da utilização do barostato de Berendsen, no valor de 1 bar, com uma constante de acoplamento de 1 ps. A pressão foi corrigida separadamente para os mesmos grupos definidos no banho térmico. 0 acoplamento de pressão para as simulações em solução aquosa foi isotrópico. A compressibilidade isotérmica foi definida como $4,5 \times 10^{-5} \mathrm{bar}^{-1}$, correspondendo ao valor próprio para o solvente escolhido na temperatura da simulação em questão.

\section{RESULTADOS E DISCUSSÃO}

Cálculos de docking molecular determinaram as principais interaçõ es entre diferentes variantes do ácido butanóico de 1,2,4-oxadiazol e a enzima HKT tendo como referência a estrutura cristalográfica do complexo HKT e inibidor competitivo ácido 4-(2-aminofenil)-4oxo-butírico (4OB) (Figura 2).

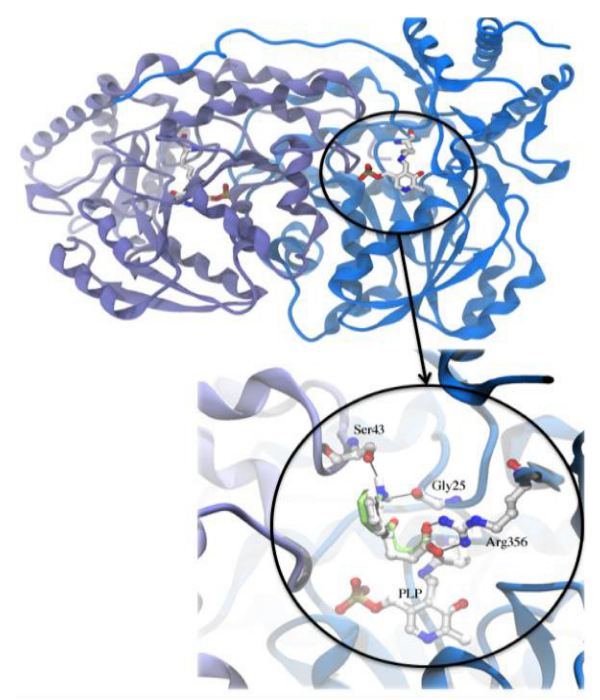

Figura 2 - Estrutura cristalográfica do complexo enzima HKT e inibidor competitivo 4OB mostrando em detalhe o cofator PLP e os resíduos envolvidos no processo de reconhecimento e inibição molecular

No presente trabalho realizamos cálculos de docking molecular para novos compostos 1,2,4-oxadiazol com maior solubilidade, e iniciamos a construção das topologias e obtenção de parâmetros atômicos para os seguintes compostos (Figura 3): o cofator piridoxal fosfato (PLP), o inibidor cristalográfico (4OB) e alguns compostos 1,2,4-oxadiazol com alta e baixa atividade inibidora da enzima HKT. Simulações de dinâmica molecular foram realizados para cada um destes compostos, e no momento estão sendo expandidas para quatro novos sistemas simulados em solvente explí cito: 1. HKT, 2. HKT-4OB, 3. HKT-OXA1 e 4. HKT-OXA2. Em todos os sistemas simulados, a enzima é tratada no estado de holoenzima. Subsequentemente, cálculos de metadinâmica serão realizados para avaliar o perfil de energia de ligação entre de cada potencial inibidor e a enzima, Discutimos através das similaridades estruturais entre as variantes do ácido butanóico de 1,2,4oxadiazol e o inibidor cristalográfico, e de valores de energia de ligação entre cada variante e a enzima qual delas tem maior potencial para ser utilizada em trabalhos de controle contra a proliferação do mosquito. Esses resultados são comparados com trabalhos anteriores a fim de validar a metodologia e confirmar tendências experimentais.
A)

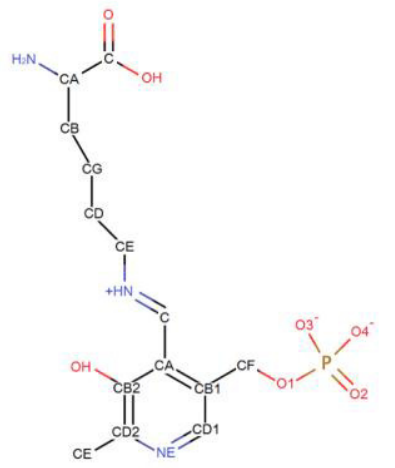<smiles>O=C(O)Cc1nc(-c2ccccc2)no1</smiles>

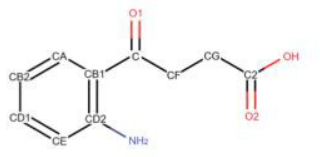

Figura 3 - Representação das topologias desenvolvidas para os compostos A) piridoxal fosfato (PLP), B) o inibidor cristalográfico (4OB) e ácido 3-(3-aril-1,2,4-oxadiazol-5-il) propiônico (protótipo para as modificações químicas objetivando desenvolvimento de moléculas com maior atividade larvicida).

\section{CONCLUSÕES}

No presente projeto foram desenvolvidas novas topologias para os compostos derivados do ácido butanóico de 1,2,4-oxadiazol, piridoxal fosfato (PLP) e para o inibidor cristalográfico (4OB), assim como foram calculados parâmetros atômicos de carga para os referidos sistemas. Simulações de dinâmica molecular para cada um dos compostos em solução atestam a adequabilidade dos novos parâmetros em solução. Uma vez tendo em mãos topologias para moléculas inexistentes no campo de força GROMOS utilizado em nossas simulações, estamos dando andamento as simulações da holoenzima na ausência e na presença de diferentes inibidores. As trajetórias resultantes destas simulações serão analisadas para comparar a dinâmica estrutural da enzima em presença de diferentes inibidores, e tendo como referencia a enzima na forma livre. As informações sobre possíveis mudanças conformacionais de pequena ou grande amplitude serão utilizadas para novos cálculos de docking molecular e para a otimização dos compostos 1,2,4oxadiazol já sintetizados em nossos grupos.

\section{REFERÊNCIAS}

MORRISON, A. C.; ZIELINSKI-GUTIERREZ, E. ; SCOTT, T. W. ; ROSENBERG, R. PLoS Med., v. 5, p. 0362-0366, 2008.

OYSTON, P.; ROBINSON, K. J. Med. Microbiol., v. 61, p. 889-894, 2012.

LIU, N.; Annu. Rev. Entomol., v. 60, p. 537-59, 2015.

LI, J.; LI, G. Insect Biochem. Mol. Biol., v. 27, p. 859-867, 1997.

LI, J. Insect Biochem. Mol. Biol., v. 29, p. 329-338, 1999.

CHEN, Y.; GUILLEMIN, G. J. Int. J. Tryptophan Res., v. 2, p. 1-19, 2009.

OLIVEIRA, V. S.; PIMENTEIRA, C. ; DA SILVA-ALVES, D. C. B.; LEAL, L. L. L.; NEVES-FILHO, R. A. W.; NAVARRO, D. M. A. F.; SANTOS, 
G. K. N.; DUTRA, K. A.; DOS ANJOS, J. V.; SOARES, T. A. Bioorg. Med. Chem., v. 21, p. 6996-7003, 2013.

MACIEL, L. G. 3-Hidróxi-quinurenina transaminase de Aedes aegypti: clonagem, expressão, avaliação funcional e síntese de prováveis inibidores seletivos. Dissertação de Mestrado em Quí mica, UFPE, Recife, 2016.

MACIEL, L. G.; ROMÃO, T. P.; LEAL, L. L. L.; SILVA-FILHA, M. H. N. L.; DOS ANJOS, J. V.; SOARES, T. A. Evidence for the role of 3 hydroxykynurenine transaminase from Aedes aegypti as a molecular target for selective inhibitors. 2017. Submitted for publication.

HAN, Q.; LI, J. FEBS Lett., v. 527, p. 199-204, 2002.

SILVA_ALVES, D. C. B.; DOS ANJOS, J. V.; CAVALCANTE, N. N. M.; SANTOS, G. K. N.; NAVARRO, D. M. A. F.; SRIVASTAVA, R. M. Bioorg. Med. Chem., v. 21, p. 940-947, 2013.

BROOIJMANS, N.; KUNTZ, I. D. Annu. Rev. Biophys. Biomol .Struct., v. 32, p. 335-73, 2003.

CHEN, L. T.; YAO, Q.; SOARES, T. A.; SQUIER, T. C.; BIGELOW, D. J. Biochemistry, v. 48, p. 2411-2421, 2009.

SOARES, T.; GOODSELL, D.; FERREIRA, R.; OLSON, A. J.; BRIGGS, J. M. J. Mol. Recogn., v. 13, p. 146-156, 2000.

SOARES, T. A.; GOODSELL, D. S.; BRIGGS, J. M.; FERREIRA, R.; OLSON, A. J. Biopolymers, v. 50, p. 319-328, 1999.

HUEY, R.; MORRIS, G. M.; OLSON, A. J.; GOODSELL, D. S. J. Comp. Chem., v. 28, p. 1145-52, 2007.

MORRIS, G. M.; HUEY, R.; LINDSTROM, W.; SANNER, M. F.; BELEW, R. K.; GOODSELL, D. S.; OLSON, A. J. J. Comp. Chem., v. 30, p. 2785-91, 2009,

SILVA, V. A.; ANDRADE, L. H. C. Etinobotânica Xucuru: espécies mí sticas. Biotemas, Florianópolis, v. 15, n. 1, p. 45-57, 2002.

GATTLI, A.; DAURA, X. VAN GUNSTEREN, W. F. Derivation of an improved simple point charge model for liquid water: SPC/A and SPC/ L. The Journal of Chemical Physics, v. 116, p. 9811-9828, 2002 